\title{
Ponderomotive effects in multiphoton pair production
}

\author{
Christian Kohlfürst* \\ Theoretisch-Physikalisches Institut, Abbe Center of Photonics, Friedrich-Schiller-Universität Jena, \\ Max-Wien-Platz, 1, D-07743 Jena, Germany \\ and Helmholtz-Institut Jena, Fröbelstieg 3, D-07743 Jena, Germany \\ Reinhard Alkofer ${ }^{\dagger}$ \\ Institute of Physics, University of Graz, NAWI Graz, Universitätsplatz, 5, 8010 Graz, Austria
}

(Received 7 December 2017; published 28 February 2018)

\begin{abstract}
The Dirac-Heisenberg-Wigner formalism is employed to investigate electron-positron pair production in cylindrically symmetric but otherwise spatially inhomogeneous, oscillating electric fields. The oscillation frequencies are hereby tuned to obtain multiphoton pair production in the nonperturbative threshold regime. An effective mass, as well as a trajectory-based semiclassical analysis, is introduced in order to interpret the numerical results for the distribution functions as well as for the particle yields and spectra. The results, including the asymptotic particle spectra, display clear signatures of ponderomotive forces.
\end{abstract}

DOI: 10.1103/PhysRevD.97.036026

\section{INTRODUCTION}

Electron-positron pair production in strong electric fields, the Sauter-Schwinger effect, is a long-standing theoretical prediction [1] which still awaits experimental verification. Multiphoton pair production, on the other hand, has already been observed in a laboratory [2]. The dynamically assisted Sauter-Schwinger effect $[3,4]$ exploits the idea that a combination of a low with a high frequency ("multiphoton") laser pulse will lead to pair production rates which are significantly larger than the sum of the rates for the two separate pulses. Together with the arising capabilities of high-intensity laser technology (see, e.g., Refs. [5,6]), such a combination of laser pulses will make experimental tests in this regime of nonlinear QED possible in the near future.

In addition to the technological progress, future experimental tests and their interpretations will depend substantially on more reliable calculations which include the inhomogeneities of the electromagnetic fields as they typically occur in the focus of crossing laser beams. Computations for inhomogeneous and time-dependent fields (i.e., beyond the previously well-studied case of time-dependent but homogeneous electric fields) began a

\footnotetext{
*hristian.kohlfuerst@uni-jena.de

reinhard.alkofer@uni-graz.at
}

Published by the American Physical Society under the terms of the Creative Commons Attribution 4.0 International license. Further distribution of this work must maintain attribution to the author(s) and the published article's title, journal citation, and DOI. Funded by SCOAP ${ }^{3}$. few years ago and have seen steady progress [7-10]. These studies are, however, still far from providing a satisfactory understanding of the effects which originate from the finite spatial extension of the considered laser pulses.

As the particle creation rate depends on the laser intensity (see, e.g., Ref. [11] for a discussion in the context of the planning of the European X-ray free-electron laser), one would naively expect that better focusing and therefore higher local field intensities always lead to an increase of the particle yield. However, the dynamics in high-intensity laser fields is much more complex and results not only in strong but also nonmonotonic dependencies of the yield on the characteristics of the laser field $[4,8,10]$. Other quantities, e.g., distribution functions and particle spectra, display a rich structure with sometimes surprisingly large sensitivities to small changes of the field parameters.

Clearly such a situation calls for the search of concepts which are able to allow an, at least qualitative, understanding. One possibility to quantify the effects associated with a spatially inhomogeneous field is based on the notion of an effective mass which an electron acquires in a background electromagnetic field [12-18]. This parameter, as every effective mass, reflects the "integrated" collective interactions of a particle with its surroundings. It thus provides the possibility of a drastic simplification but nevertheless allows the coarse-grained description of highly intricate effects. Although this might be an oversimplification with respect to details of the resulting spectra, the concept of an effective mass works astonishingly well, a fact which can be attributed to the unique conditions in high-intensity laser experiments [19]. Correspondingly, the idea of an effective mass has been 
applied recently to multiphoton pair production [20,21]. In these studies, however, the employed electric fields were homogeneous, thereby greatly simplifying the process under investigation.

In the following we will discuss particle creation in inhomogeneous fields and introduce, to this end, a more general concept for the effective mass and relate it via a semiclassical analysis to ponderomotive forces. (NB: A ponderomotive force is a nonlinear force that a "classical" charged particle experiences in an inhomogeneous oscillating electromagnetic field, cf. Ref. [22] and references therein.) In a first step, we will concentrate on multiphoton pair production in the nonperturbative threshold regime. As is evident from the discussion above, in the parameter regions of interest the effects of a laser pulse's finite spatial extension cannot be neglected. (For a more detailed discussion of this issue, see, e.g., Ref. [23].) Hereby one should distinguish two aspects: First, how does the finiteness of the fields' extension influence the pair production process, and second, how does a spatially inhomogeneous field alter the subsequent electron/positron dynamics? To keep the calculational complexity in a manageable range, we will assume cylinder symmetry of the electric field. The spatial dependence can be inferred, e.g., from a gauge potential which points in one direction and is inhomogeneous only w.r.t. the same direction. This has the advantage that the corresponding magnetic field vanishes. However, one then clearly has no propagating waves. Nevertheless, such a configuration provides some, although simplistic, model of the focus of two counterpropagating lasers.

This paper is organized as follows. In Sec. II several aspects of multiphoton pair production in the nonperturbative threshold regime underlying our work will be briefly summarized: In Sec. II A a short description of the DiracHeisenberg-Wigner (DHW) formalism for the case of an electric field with cylinder symmetry is provided; Sec. II B deals with the numerical treatment via Fourier transform; in Sec. II C the form of the electric field employed later is given. In Sec. III two interpretational concepts are introduced and discussed: the effective mass and a trajectorybased semiclassical analysis. In Sec. IV numerical results, ordered according to the longitudinal and transverse momentum distributions, are presented and discussed. Section V contains the conclusions of the presented study.

Furthermore, we use $\hbar=c=1$ throughout this paper.

\section{THEORETICAL DESCRIPTION OF MULTIPHOTON PAIR PRODUCTION}

\section{A. DHW formalism}

The present study is based on the DHW formalism, a relativistic phase-space approach, which has been developed for the case of pair production in Ref. [24]. Hereby the electron is treated as a quantum field, but the laser pulse is approximated by its mean field. Given the magnitudes of the electric field needed in pair production, this is a justified approximation.

A convenient starting point is provided by the gaugeinvariant density operator of the system,

$$
\hat{\mathcal{C}}_{\alpha \beta}(r, s)=\mathcal{U}(A, r, s)\left[\bar{\psi}_{\beta}(r-s / 2), \psi_{\alpha}(r+s / 2)\right],
$$

in terms of the electron's spinor-valued Dirac field $\psi_{\alpha}(x)$. Hereby $r$ denotes the center of mass and $s$ the relative coordinate. The Wilson line factor

$$
\mathcal{U}(A, r, s)=\exp \left(\mathrm{i} e s \int_{-1 / 2}^{1 / 2} d \xi A(r+\xi s)\right)
$$

is introduced to render the density operator gauge invariant. It depends on the elementary charge $e$ and the background gauge field $A$. Treating the background field in the Hartree approximation, i.e.,

$$
F^{\mu \nu}(x) \approx\left\langle\hat{F}^{\mu \nu}(x)\right\rangle
$$

no path ordering is needed, and in a given Lorentz frame and gauge, the background gauge field $A(\mathbf{x}, t)$ is a fixed $c$-number valued function. The covariant Wigner operator

$$
\hat{\mathcal{W}}_{\alpha \beta}(r, p)=\frac{1}{2} \int d^{4} s \mathrm{e}^{\mathrm{i} p s} \hat{\mathcal{C}}_{\alpha \beta}(r, s)
$$

thus reflects the quantum fluctuations of the electron but not the one of the laser field.

The main implication of the Hartree approximation for the electromagnetic field becomes evident when taking the vacuum expectation value of the covariant Wigner operator to obtain the covariant Wigner function

$$
\mathbb{W}(r, p)=\langle\Phi|\hat{\mathcal{W}}(r, p)| \Phi\rangle .
$$

In its equation of motion the electromagnetic field can be factored out, e.g.,

$$
\left\langle\Phi\left|F_{\mu \nu} \hat{\mathcal{C}}\right| \Phi\right\rangle=F_{\mu \nu}\langle\Phi|\hat{\mathcal{C}}| \Phi\rangle
$$

which allows us to resolve the otherwise infinite Bogoliubov-Born-Green-Kirkwood-Yvon hierarchy of correlation functions.

Being a Dirac-matrix valued quantity, the Wigner function is best decomposed into 16 covariant Wigner coefficients

$$
\mathbb{W}=\frac{1}{4}\left(\mathbb{1} \mathbb{S}+\mathrm{i} \gamma_{5} \mathbb{P}+\gamma^{\mu} \mathbb{V}_{\mu}+\gamma^{\mu} \gamma_{5} \mathbb{A}_{\mu}+\sigma^{\mu \nu} \mathbb{T}_{\mu \nu}\right)
$$

where the corresponding transformation properties are made evident by the notation. Working in a definite frame it proves advantageous to project on equal times, which yields the equal-time Wigner function 


$$
\mathbb{W}(\mathbf{x}, \mathbf{p}, t)=\int \frac{d p_{0}}{2 \pi} \mathbb{W}(r, p)
$$

and, by an analogous decomposition to Eq. (7), the corresponding equal-time Wigner coefficients $\mathbb{s}, \mathbb{p}, \mathbb{v}_{0, x, y, z}, \ldots$

Exploiting cylindrical symmetry, which will be assumed throughout the following, and keeping the electric field homogeneous in the transversal direction results in a reduced system of differential equations ${ }^{1}$ :

$$
\begin{gathered}
D_{t} \overline{\mathrm{s}}-2 p_{z} \overline{\mathrm{P}}+2 p_{\rho} \overline{\mathrm{v}}=0, \\
D_{t} \overline{\mathrm{v}}+\partial_{z} \overline{\mathrm{v}}_{0}-2 p_{\rho} \overline{\mathrm{s}}=-2 \overline{\mathrm{p}}, \\
D_{t} \overline{\mathrm{P}}^{\mathrm{P}}+2 p_{z} \overline{\mathrm{s}}=2 \overline{\mathrm{v}}, \\
D_{t} \overline{\mathrm{v}}_{0}+\partial_{z} \overline{\mathrm{v}}=0,
\end{gathered}
$$

where the pseudodifferential operator $D_{t}$ reads

$$
D_{t}=\partial_{t}+e \int d \xi E\left(z+\mathrm{i} \xi \partial_{p_{z}}, t\right) \partial_{p_{z}} .
$$

We refrained from putting a spatial index on the electric field $E$ as, by construction, it is oriented in the $z$-direction.

The advantage of a representation with Wigner coefficients lies in the fact that it allows us to identify $\bar{s}$ as mass, $\overline{\mathrm{v}}_{0}$ as charge, and $\overline{\mathrm{v}}$ as current density [24]. In order to perform calculations within the DHW formalism, we employ vacuum initial conditions:

$$
\overline{\mathrm{s}}_{\text {in }}=-\frac{2}{\omega}, \quad \overline{\mathbb{v}}_{\text {in }}=-\frac{2 p_{z}}{\omega}, \quad \overline{\mathbb{P}}_{\text {in }}=-\frac{2 p_{\rho}}{\omega},
$$

where $\omega=\omega\left(p_{z}, p_{\rho}\right)=\sqrt{m^{2}+p_{z}^{2}+p_{\rho}^{2}}$ is the one-particle energy. It is convenient to subtract these initial conditions, and therefore we define

$$
\overline{\mathbb{W}}^{v}:=\overline{\mathbb{W}}-\overline{\mathbb{W}}_{\text {in }},
$$

where $\overline{\mathbb{W}}$ is a placeholder for any Wigner component.

Additionally, we define the particle number density per unit volume in momentum space,

$$
N\left(p_{z}, p_{\rho}\right)=\int d z \frac{\overline{\mathbb{s}}^{v}+p_{z} \overline{\mathbb{v}}^{v}+p_{\rho} \overline{\mathbb{p}}^{v}}{\omega\left(p_{z}, p_{\rho}\right)} .
$$

Consequently, the total particle yield per unit volume is defined via

$$
N=\int d p_{z} \int d p_{\rho} N\left(p_{z}, p_{\rho}\right)
$$

\footnotetext{
${ }^{1}$ The coefficients given in the following are obtained by linear combinations of the equal-time Wigner coefficients. The quantity $\bar{v}$, for example, is defined as a linear superposition of $\mathbb{v}_{z}$ and tensor components $\mathbb{t}_{x z}$ and $\mathbb{t}_{y z}$. The details of the derivation can be found in Ref. [23].
}

\section{B. Fourier transform and numerical treatment}

Equations (9)-(12) can be solved numerically without any further approximations or truncations [8,23]. The challenging part is the nonlocality of the pseudodifferential operator (13) which can nevertheless be treated if the electric field $E(z, t)$ can be Taylor expanded and integrated with sufficient accuracy. The differential operator (13) naturally splits into two parts:

$$
D_{t}=\partial_{t}+e \int d \xi E\left(z+\mathrm{i} \xi \partial_{p_{z}}, t\right) \partial_{p_{z}}=: \partial_{t}+\Delta .
$$

To apply the operator $\Delta$ on the (subtracted) Wigner components, we Fourier transform and inverse Fourier transform $\Delta \overline{\mathbb{W}}^{v}$ w.r.t. to the variable $p_{z}$; i.e., we employ

$$
f\left(p_{z}\right)=\mathcal{F}_{p_{z}}^{-1}\left[\mathcal{F}_{p_{z}}\left[f\left(p_{z}\right)\right]\right]=\mathcal{F}_{p_{z}}^{-1}\left[\tilde{f}\left(\mathrm{k}_{p_{z}}\right)\right],
$$

Taylor expand the electric field, use

$$
\mathcal{F}_{p_{z}}\left[\frac{d^{n}}{d p_{z}^{n}} f\left(p_{z}\right)\right]=\left(\mathrm{ik}_{p_{z}}\right)^{n} \tilde{f}\left(\mathrm{k}_{p_{z}}\right),
$$

and then resum to obtain the generic form (for more details, see Ref. [23])

$$
\begin{aligned}
& \Delta \overline{\mathbb{W}}^{v}\left(z, p_{z}, t\right) \\
& \quad=\mathcal{F}_{p_{z}}^{-1}\left[\operatorname{iek}_{p_{z}} \int d \xi E\left(z-\xi \mathrm{k}_{p_{z}}, t\right) \tilde{\overline{\mathbb{W}}}\left(z, \mathrm{k}_{p_{z}}, t\right)\right] .
\end{aligned}
$$

Due to the fact that a Fourier transform takes into account all points in a domain, the introduction of global basis functions turned out to be favorable compared to the finitedifference method used in Ref. [8]. Hence, we have equidistantly discretized spatial and momentum directions, respectively, turning the system of partial differential equations (9)-(12) into a high-dimensional $\left(N_{z} \times N_{p_{\rho}} \times N_{p_{z}}\right)$ system of ordinary differential equations with $t$ as the only continuous parameter.

Furthermore, we choose periodic boundary conditions in $z$ and $p_{z}$,

$$
\overline{\mathbb{N}}^{v}\left(z_{0}, p_{z}, t\right)=\overline{\mathbb{W}}^{v}\left(z_{N_{z}}, p_{z}, t\right)
$$

and

$$
\overline{\mathbb{W}}^{v}\left(z, p_{z, 0}, t\right)=\overline{\mathbb{W}}^{v}\left(z, p_{z, N_{p_{z}}}, t\right),
$$

respectively. Additionally, we set

$$
\overline{\mathbb{W}}^{v}\left(z_{k_{i}}, p_{z, k_{j}}, t\right)=0 \quad \text { if } k_{i}=0 \quad \text { or } \quad k_{j}=0 .
$$

These choices do not influence the numerical results as long as the chosen discretized domain is sufficiently large, 
and the number of grid points in every direction is sufficiently high.

After discretizing Eqs. (9)-(12) in this way, they can be solved using pseudospectral methods [25]. The time integration is done using a Dormand-Prince Runge-Kutta integrator of order $8(5,3)$ [26].

\section{Model for the fields}

As stated above, if the spatial dependence of the electric field is inferred from a gauge potential which is directed along a direction and is inhomogeneous only w.r.t. the same direction, the corresponding magnetic field vanishes. In addition, two time scales are needed to tune a pulse of finite duration to the multiphoton regime. These requirements are fulfilled by the ansatz

$$
\begin{aligned}
E(z, t) & =\varepsilon E_{0} \mathcal{E}(z) F(t) \\
& =\varepsilon E_{0} \mathcal{E}(z) \cos ^{4}\left(\frac{t}{\tau}\right) \cos (\omega t),
\end{aligned}
$$

for $t \in[-\pi \tau / 2, \pi \tau / 2]$, and $E=0$ otherwise (see Fig. 1). Hereby the critical field strength $E_{0}=m^{2} / e$ has been factorized out for convenience. Nonperturbative multiphoton pair production is probed if one chooses the product $\omega \tau>1$ and a Keldysh parameter of $\gamma=\omega / m \varepsilon>1$ [23].

As we want to investigate how focusing influences the particle distribution rate, we choose a well-localized electric field with a Gaussian shape of width $\lambda$ :

$$
\mathcal{E}(z)=\exp \left(-\frac{z^{2}}{2 \lambda^{2}}\right) .
$$

In Fig. 2 we sketch the electric field for fixed times. Note that the electric field varies in strength but always points in the $\pm z$-direction.

The model proposed in Eqs. (25) and (26) may be thought of as field generated in an antinode of a standing-wave

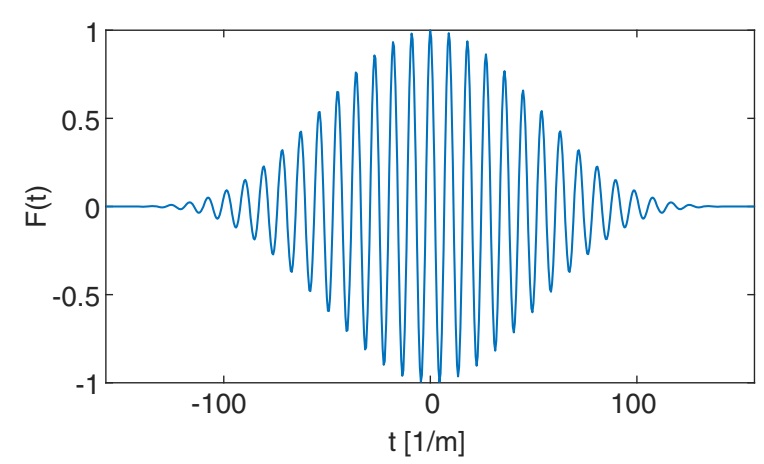

FIG. 1. Sketch of the time-dependent part of the electric field model $F(t)$ for $\tau=100 \mathrm{~m}^{-1}$ and $\omega=0.7 \mathrm{~m}$. The large number of cycles $(\omega \tau=70 \gg 1)$ is an indicator for the nonperturbative multiphoton regime.

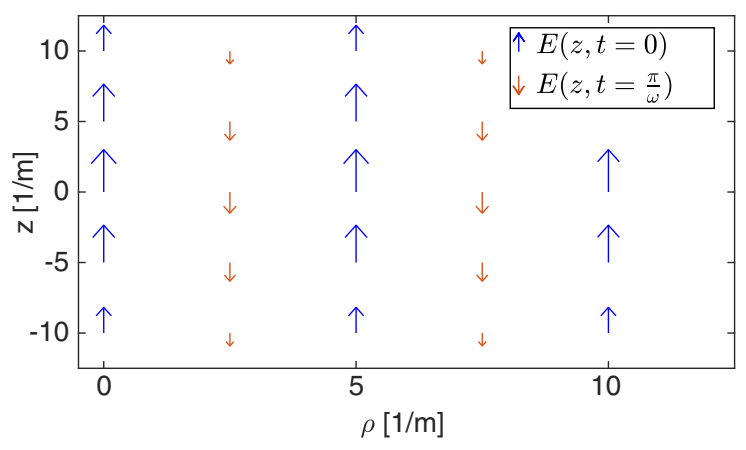

FIG. 2. Illustration of the electric field as a function of the spatial coordinates $z$ and $\rho$ for fixed time $t=0$ (blue) and $t=\frac{\pi}{\omega}$ (red). Due to the special geometry of the model, the field is homogeneous in the transversal direction.

mode. As a special feature, this model takes into account spatial variations besides time dependence.

\section{SEMICLASSICAL ANALYSIS}

In Sec. IV it will become obvious that the dependencies of observables on the field parameters are of an astonishing complexity. In order to obtain an interpretation and such an understanding of the results, we will analyze them by referring to the concepts developed in this section.

Introducing a generalized effective mass concept and relating it to arising ponderomotive forces are central aspects of this paper. We discuss the improvements compared to previous definitions of the effective mass in the context of pair production in Sec. III A. Moreover, based upon Refs. [22,27], we draw a connection between a spatially dependent effective mass and ponderomotive forces. However, an interpretation of the final particle distribution on the basis of an effective mass becomes more involved for spatially inhomogeneous fields due to the position dependence of the gradient. Hence, we rely on a semiclassical trajectory-based model, which allows us to determine the overall scheme by simple means.

\section{A. Effective mass and ponderomotive forces}

Various studies have used effective masses to simplify intermediate calculations $[14,15,28,29]$. It was suggested only recently to employ the concept of an effective mass to determine directly observable quantities regarding particle creation (see Refs. [20,30]).

In the case of a monochromatic plane wave, the effective mass takes the form [12]

$$
M_{*}=m \sqrt{1+\xi^{2}}, \quad \text { with } \quad \xi=\frac{e}{m} \sqrt{-\left\langle A^{\mu} A_{\mu}\right\rangle} .
$$

More general definitions have been proposed in Ref. [29]. However, we essentially adopt but modify slightly the definition above and parametrize the effective mass as follows: 


$$
\begin{aligned}
m_{*}(\mathbf{x}) & =m \sqrt{1+\tilde{\xi}(\mathbf{x})^{2}}, \\
\tilde{\xi}(\mathbf{x}) & =\frac{e}{m} \sqrt{-\left\langle A_{\mu}(\mathbf{x}, t) A^{\mu}(\mathbf{x}, t)\right\rangle} .
\end{aligned}
$$

Similarly to Refs. [20,29], we cope with the temporal finiteness of the pulse by averaging over one field oscillation around $t=0$ only; this approximation is well justified for long, flat-topped multicycle pulses due to the minor influence of the envelope function. ${ }^{2}$

The relativistic ponderomotive force then yields (see Ref. [22])

$F_{p}=-\left(\mathbf{v}_{0} \cdot \nabla_{x} m_{*}, \nabla_{x} m_{*}+\frac{\gamma_{0}-1}{v_{0}^{2}}\left(\mathbf{v}_{0} \cdot \nabla_{x} m_{*}\right) \mathbf{v}_{0}\right)$,

where $\gamma_{0}$ is the Lorentz factor and $\mathbf{v}_{0}$ denotes the velocity of the quasiparticle.

We do not use Eq. (29) directly to calculate reference values. However, as Eq. (29) describes an effective force, where all short-scale contributions are "integrated out," it primarily serves as a tool to help us interpret the results obtained from solving the system (9)-(12). Analyzing Eq. (29) analytically, we can deduce that the term $\boldsymbol{\nabla}_{x} m_{*}$ is the decisive factor in order to understand the effective force on the particles. In turn, we expect that all particles are forced from strong-field towards weak-field regions. Furthermore, in the case under consideration we primarily expect a boost in the parallel momentum. If particles are created with vanishing transversal momentum, ponderomotive forces only act upon them in a direction parallel to the applied field.

\section{B. Trajectory-based semiclassical model}

The virtue of the DHW method, namely, to take into account various effects in one common approach, obstructs the analysis of its results. Hence, we introduce a trajectorybased model in order to overcome these difficulties.

Contrary to Schwinger pair production the formation time in multiphoton pair production is quite long. For the sake of simplicity, we still want to assume that particles are created at points in space-time where the electric field takes on its maximal values. ${ }^{3}$ Moreover, for the case of $n$-photon pair production we expect that the initial particle momenta $p$ can be derived via energy conservation laws $[20,31]$ :

\footnotetext{
${ }^{2}$ In Ref. [30] the effective mass was introduced via a "renormalized frequency" (Fourier zero mode) for purely timedependent fields. Although defined differently it was shown that it agrees very well with $m_{*}(\mathbf{x}=0)$.

${ }^{3}$ We are well aware of the fact that this assumption leads to an oversimplification of the description of multiphoton processes as particles can be created at all times. However, evaluating particle trajectories that start at turning points of the applied fields yields reference values for the final particle momenta, which suffices, in the context of the current investigation, to validate the method.
}

$$
p_{n}^{2}=\left(\frac{n \omega}{2}\right)^{2}-m_{*}^{2}
$$

These assumptions are sufficient to determine the trajectory of a particle in an external field as they provide all needed initial conditions.

Due to the form of the electric field and especially due to the absence of a magnetic field, spin effects will be ignored at this point. Hence, we employ the relativistic Lorentz equation

$$
\frac{d P^{\mu}}{d \tau}=e F^{\mu \nu} U_{\nu}
$$

to analyze the particle's trajectory in the external field. Here, $F^{\mu \nu}$ is the electromagnetic field strength tensor, $U_{\nu}$ the four-velocity and $P^{\mu}$ the four-momentum.

This method is a convenient yet powerful tool to analyze the dynamics of pair production. However, it should be understood as a simple approximation, which clearly cannot replace a full quantum field theoretical treatment of the process. In Ref. [23] its usefulness is demonstrated for a variety of field configurations.

\section{NUMERICAL RESULTS FOR MULTIPHOTON PAIR PRODUCTION IN THE THRESHOLD REGIME}

In the following we discuss the solutions of the PDEs (9)-(12) and compare the outcome with the results obtained from the trajectory-based approach. We analyze the distribution function for parallel particle momenta and, subsequently, the total production rate.

For the pulse length we have chosen a value of $\tau=$ $100 \mathrm{~m}^{-1}$ for all calculations. Such a pulse length is sufficient to capture all essential features of multiphoton pair production.

\section{A. Parallel momentum distribution}

In this subsection we only analyze particle spectra where $p_{\rho}=0$. In Fig. 3 a typical spectrum for multiphotondominated particle creation is displayed. Especially for quasihomogeneous fields, $\lambda=1000 \mathrm{~m}^{-1}$, the characteristic multiphoton peaks are easily distinguishable.

To determine the contributing processes we can employ the effective mass picture [20]. In the case of spatially slowly varying fields, the vector potential may be treated as homogeneous. Hence, ignoring the spatial dependence in Eq. (25), we can determine the particle's effective mass (28),

$$
m_{*} \approx m \sqrt{1+\frac{\varepsilon^{2} m^{2}}{2 \omega^{2}}} .
$$

Employing Eq. (30) the particle momenta can be estimated as

$$
p_{z, n} \approx \sqrt{\left(\frac{n \omega}{2}\right)^{2}-m^{2}\left(1+\frac{\varepsilon^{2} m^{2}}{2 \omega^{2}}\right)} .
$$




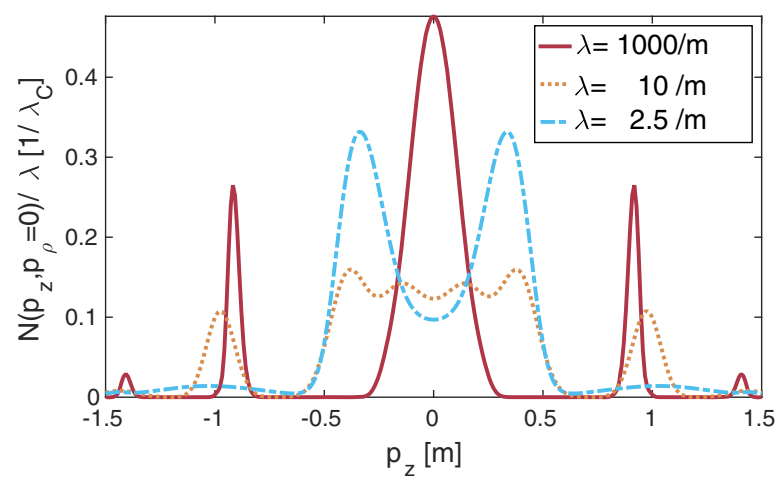

FIG. 3. Reduced particle distribution function obtained from a DHW calculation for a field of strength $\varepsilon=0.5$, length $\tau=$ $100 \mathrm{~m}^{-1}$ and frequency $\omega=0.7 \mathrm{~m}$. The peaks can be related to $n$-photon pair production. The stronger the focus, the stronger the ponderomotive forces, and thus the higher the final particle momentum.

From this relation it is easy to infer that the peak in the middle is related to a 3-photon process (threshold frequency $\tilde{\omega}=0.74 \mathrm{~m}$ ) and the side maxima are related to 4 - and 5-photon pair production.

However, comparing the relative peak sizes with results obtained from calculations within homogeneous fields, one finds that the strong peak around $p_{z}=0$ is now more pronounced. Nevertheless, we recover the quantum kinetic limit [32] when evaluating the results for $\lambda \rightarrow \infty$ at $z=0$.

Going beyond this limit reveals that a decrease of the pulse's spot size leads to dramatic changes in the distribution function. In the case of, e.g., $\lambda=10 \mathrm{~m}^{-1}$, the dominant peak in the particle momentum spectrum takes on a much wider form which can be related to quantum interferences. It is known from atomic ionization [33] that $n$-photon peaks can be interpreted as a result of particle trajectories adding up to the interference pattern observable [34]. Around $\lambda=10 \mathrm{~m}^{-1}$ the finite size of the laser pulse seems to prevent a coherent superposition. In turn, the corresponding interference pattern becomes disturbed, resulting in the broadened distribution. A quantitative comparison between the trajectory-based approach and the DHW formalism is summarized in Table I.

At an even smaller focus size, $\lambda=2.5 \mathrm{~m}^{-1}$, we observe so-called peak splitting. This effect can be understood in the context of ponderomotive forces. Concentrating on the 3-photon peak, particles are created with close to vanishing momentum $p_{z}$ and subsequently follow the oscillations of the background field. However, the force the field exerts on the particle depends on the particle's location in the field. The momentum the particle acquires in one half-cycle within the strong field region of the field cannot be fully compensated within the second half-cycle because the particle has already entered the weak-field region. As a result the particle obtains a net momentum and drifts to low-intensity regions in space. As the applied electric field is symmetric in $z$, there are, however, two equally likely
TABLE I. Trajectory analysis of particles within an external field. The results are obtained by evaluating the relativistic Lorentz force equation for particles seeded at $t_{0}=0, z_{0}=0$ in a field of strength $\varepsilon=0.5$, length $\tau=100 \mathrm{~m}^{-1}$, frequency $\omega=0.7 \mathrm{~m}$ and spatial extent $\lambda$. The different initial momenta $p_{z, 0}$ correspond to different $n$-photon processes. The final momenta $p_{z, f}$ are obtained at asymptotic times. For comparison we provide the results from a DHW calculation $p_{\mathrm{DHW}}$.

\begin{tabular}{lccc}
\hline \hline$\lambda\left[\mathrm{m}^{-1}\right]$ & $p_{z, 0}[\mathrm{~m}]$ & $p_{z, f}[\mathrm{~m}]$ & $p_{\text {DHW }}[\mathrm{m}]$ \\
\hline 1000 & 0 & $10^{-7}$ & 0 \\
10 & 0 & 0.162 & $0-0.38$ \\
2.5 & 0 & 0.444 & 0.33 \\
1000 & 0.92 & 0.92 & 0.92 \\
10 & 0.92 & 0.99 & 0.98 \\
2.5 & 0.92 & 1.12 & 1.03 \\
1000 & 1.4 & 1.4 & 1.40 \\
10 & 1.4 & 1.43 & 1.43 \\
2.5 & 1.4 & 1.65 & 1.45 \\
\hline \hline
\end{tabular}

options: Either the particles are accelerated in the $z$ or in the $-z$ direction. As a result, the two peaks at nonzero final momentum and the valley at $p_{z}=0$ are formed.

Particles with nonzero initial momenta acquire only an additional push due to ponderomotive forces. The strength of this boost depends on the initial conditions but seems to be nonmonotonic following the results in Table I. We interpret the increase in momentum as a consequence of particle acceleration out of the strong field region within one half-cycle. If the spatial extent of the background field is sufficiently small, these particles are basically unaffected by the following field oscillations and keep their respective momentum.

A finite spatial extent also affects the particle production rate. Figure 4 shows the reduced particle yield for different photon frequencies and various spot sizes. In the case of 3-photon pair production (blue line in Fig. 4), the introduction of a spatial extent only lowers the particle production rate.

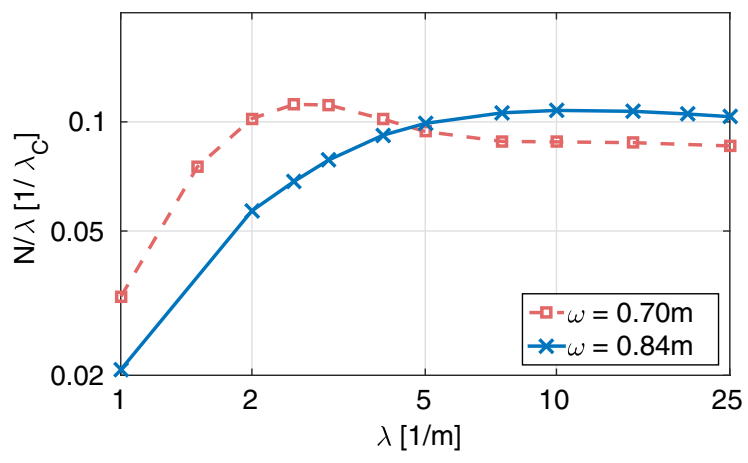

FIG. 4. Log-log plot of the reduced particle yield $N / \lambda$ calculated from the DHW equations for various field frequencies $\omega$ as a function of the spatial extent $\lambda$. The sharp decrease for $\lambda \sim \mathcal{O}(1)$ is directly related to the lack of sufficient field energy to produce particles. Parameters: $\varepsilon=0.5, \tau=100 \mathrm{~m}^{-1}$. 
However, in the case of photon frequencies of $\omega=0.7 \mathrm{~m}$ and a field strength of $\varepsilon=0.5$, a 3 -photon pair production process is not possible due to energy conservation laws; see Eq. (33) and Ref. [20]. The fact that we still observe a peak at $p_{z} \sim 0$ in the particle spectrum (see Fig. 3 ) is a hint towards a dynamically assisted tunneling process, where absorbing 3 photons lowers the energy barrier and, in turn, increases the likelihood of a tunneling process to happen. Additionally, the 4-photon creation process contributes towards the yield in Fig. 4 (dashed red line). All in all, it seems as if especially particles created via the assistance mechanism [4] benefit from a small spot size and the resulting strong ponderomotive forces leading to an overall increase in the reduced yield. Concerning the drop-off for small values of the spatial extent $\lambda$ : If the spatial extension of the field is of the order of the Compton wavelength, the total electric field energy becomes too small to produce a sizable amount of particles [8].

\section{B. Transversal momentum distribution}

Following the discussion on particle acceleration parallel to the applied field, we now turn our attention towards electrons/positrons, which are created with nonvanishing transversal momentum. For demonstration purposes we have chosen a slightly higher field frequency. In this way, the implications of ponderomotive forces in the particle momentum spectrum become more evident. The Keldysh parameter for the field used is $\gamma=1.68$, indicating a process in the crossover regime with multiphoton dominance [23].

The comparison between a flat $\left(\lambda=100 \mathrm{~m}^{-1}\right)$ and a sharp peak $\left(\lambda=2 \mathrm{~m}^{-1}\right)$ is illustrated in Fig. 5 with focus on a 3-photon process. The figure at the top shows a situation close to a result obtained via a purely timedependent electric field [21,35]. Particles created via photon absorption obtain a well-defined total momentum (30). Additionally, the resulting ringlike structure is superimposed by quantum interferences $[23,31]$.

A smaller spatial extension of the laser focus leads to an increase in strength of ponderomotive forces, clearly visible in Fig. 5 in the form of an acceleration in the $p_{z}$-direction. Similar to the previous case, the bunch of particles at $p_{z}=0, p_{\rho}=0.7 \mathrm{~m}$ is boosted in either $p_{z}$-direction, leading to peak splitting. Moreover, the interference pattern diminishes, and the sharp peaks signalizing $n$-photon processes are washed out. Once again, we analyze the particle trajectories to understand this line broadening. Constraining ourselves to the center of the laser pulse, we seed the particles at local maxima of the applied electric field and calculate the particles' final momenta in dependence of the spatial extent; see Table II. In the case of a broad spatial extent, all particles acquire the same final momenta. In the case of a small extent, however, the finiteness of the pulse plays a crucial role because then different particle creation times lead to different behavior.
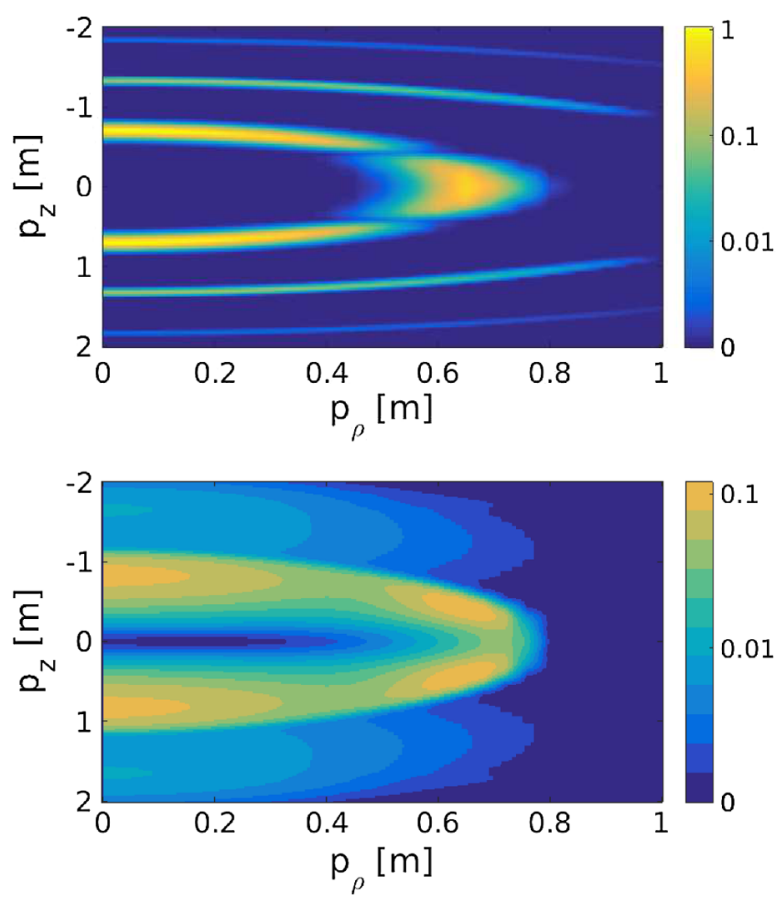

FIG. 5. Momentum maps $N / \lambda$ obtained from evaluating the DHW equations. The electric field features a field strength of $\varepsilon=0.5$, a pulse length of $\tau=100 \mathrm{~m}^{-1}$, a field frequency of $\omega=$ $0.84 \mathrm{~m}$ and spatial extents $\lambda=100 \mathrm{~m}^{-1}$ (top) or $\lambda=2 \mathrm{~m}^{-1}$ (bottom). The additional acceleration in the parallel direction (in the second plot) can be attributed to strong ponderomotive forces. The result is line broadening and peak splitting.

Due to the fact that we seed the particles at maxima of the field only, we obtain an upper and a lower limit for the particles' final momenta. Assuming that electrons and positrons are created also at intermediate times, we expect that their respective final momenta are distributed in between these two limits. It is therefore not surprising that the peaks in the momentum spectrum appear much wider compared to the quasihomogeneous case.

The smaller boost for higher transversal momenta is a consequence of relativistic mechanics as can be seen by comparison with the effective ponderomotive forces (29).

TABLE II. The relativistic Lorentz force equation is evaluated for particles seeded at local maxima of the field, $t_{0}$ and $z_{0}=0$, in a field of strength $\varepsilon=0.5$, length $\tau=100 \mathrm{~m}^{-1}$, frequency $\omega=$ $0.84 \mathrm{~m}$ and spatial extent $\lambda$. The initial momenta were chosen to be $p_{z, 0}=0.665 \mathrm{~m}$ and $p_{\rho, 0}=0$, resembling the photon peak positions in Fig. 5.

\begin{tabular}{lcc}
\hline \hline$t_{0}\left[\mathrm{~m}^{-1}\right]$ & $p_{z, f}(\lambda=100)[\mathrm{m}]$ & $p_{z, f}(\lambda=2)[\mathrm{m}]$ \\
\hline$-2 \pi / \omega$ & 0.70 & 0.79 \\
$-\pi / \omega$ & 0.70 & 0.85 \\
0 & 0.70 & 0.80 \\
$\pi / \omega$ & 0.70 & 0.84 \\
$2 \pi / \omega$ & 0.70 & 0.80 \\
\hline \hline
\end{tabular}


TABLE III. Evaluating the relativistic Lorentz force equation for particles seeded at $t_{0}=0, z_{0}=0$ with parallel momentum $p_{z, 0}=0$ and transversal momentum $p_{\rho, 0}$. The external field shows a strength of $\varepsilon=0.5$, a pulse length of $\tau=100 \mathrm{~m}^{-1}$ and a frequency of $\omega=0.7 \mathrm{~m}$. The spatial extent $\lambda$ is varied. The final parallel momenta $p_{z, f}$ are obtained at asymptotic times. Note that the transversal momenta are nearly unaffected by the external field.

\begin{tabular}{lcc}
\hline \hline$p_{\rho, 0}[\mathrm{~m}] \sim p_{\rho, f}[\mathrm{~m}]$ & $\lambda\left[\mathrm{m}^{-1}\right]$ & $p_{z, f}[\mathrm{~m}]$ \\
\hline 0 & 1000 & $8 \times 10^{-6}$ \\
0.5 & 1000 & $7 \times 10^{-6}$ \\
1 & 1000 & $4 \times 10^{-6}$ \\
0 & 20 & 0.026 \\
0.5 & 20 & 0.021 \\
1 & 20 & 0.013 \\
0 & 10 & 0.16 \\
0.5 & 10 & 0.12 \\
1 & 10 & 0.07 \\
\hline \hline
\end{tabular}

For the sake of completeness, we provide the data obtained through a trajectory analysis in Table III.

\section{CONCLUSIONS}

We have presented numerical solutions describing multiphoton pair production for oscillating, spatially inhomogeneous electric fields in the DHW formalism. Spatial inhomogeneities introduce effective ponderomotive forces, which directly affect the particle momentum distribution and subsequently the total yield. Moreover, we have shown that these forces can be understood via a generalized effective mass concept.
With the aid of a semiclassical trajectory-based model, we produced reference values to analyze our findings regarding new phenomena connected with a finite spatial pulse size: peak splitting and line broadening. As for the first effect, we note that the peaks split due to strong ponderomotive forces altering the particle momentum spectrum. Line broadening happens because the particle spectra properties which are characteristic for multiphoton pair production erode, and instead of sharp lines one obtains broad bunches.

In summary, we presented here further evidence for how important it is to take spatial inhomogeneities of the fields underlying pair production processes into account. Therefore, further investigations aiming at an understanding of matter creation from fields will at least have to include spatial variations of the fields. Given sufficient computational resources the DHW formalism can be readily extended such that inhomogeneous magnetic fields can be included. This would imply a major step towards understanding multiphoton pair production in realistic scenarios, further closing the gap between theory and experiment.

\section{ACKNOWLEDGMENTS}

We thank T. Heinzl for helpful and interesting discussions. C. K. acknowledges financial support by the FWF (Austrian Science Fund) Doctoral Program DK W1203-N16, by the BMBF under Grant No. 05P15SJFAA (FAIR-APPASPARC), and by the Helmholtz Association through the Helmholtz Postdoc Programme (PD-316). Computations were performed on the "Supermicro Server 1028TR-TF" in Jena, which was funded by the Helmholtz Postdoc Programme (PD-316).
[1] F. Sauter, Z. Phys. 69, 742 (1931); W. Heisenberg and H. Euler, Z. Phys. 98, 714 (1936); J. S. Schwinger, Phys. Rev. 82, 664 (1951).

[2] D. L. Burke et al. (SLAC Collaboration), Phys. Rev. Lett. 79, 1626 (1997); C. Bamber et al., Phys. Rev. D 60, 092004 (1999).

[3] R. Schützhold, H. Gies, and G. Dunne, Phys. Rev. Lett. 101, 130404 (2008); M. Orthaber, F. Hebenstreit, and R. Alkofer, Phys. Lett. B 698, 80 (2011); B. King, H. Gies, and A. Di Piazza, Phys. Rev. D 86, 125007 (2012); T. Nousch, D. Seipt, B. Kampfer, and A. I. Titov, Phys. Lett. B 715, 246 (2012); I. Akal, S. Villalba-Chávez, and C. Müller, Phys. Rev. D 90, 113004 (2014); A. Blinne and H. Gies, Phys. Rev. D 89, 085001 (2014); A. Blinne and E. Strobel, Phys. Rev. D 93, 025014 (2016); F. Fillion-Gourdeau and S. MacLean, Phys. Rev. B 92, 035401 (2015); A. Otto, D. Seipt, D. Blaschke, S. A. Smolyansky, and B. Kämpfer, Phys. Rev. D 91, 105018
(2015); A. Ilderton, G. Torgrimsson, and J. Wårdh, Phys. Rev. D 92, 025009 (2015); A. Blinne, Ph.D. Thesis, University of Jena, 2016; C. Schneider and R. Schützhold, Phys. Rev. D 94, 085015 (2016); G. Torgrimsson, J. Oertel, and R. Schützhold, Phys. Rev. D 94, 065035 (2016).

[4] M. F. Linder, C. Schneider, J. Sicking, N. Szpak, and R. Schützhold, Phys. Rev. D 92, 085009 (2015).

[5] T. Heinzl and A. Ilderton, Eur. Phys. J. D 55, 359 (2009).

[6] M. Marklund and J. Lundin, Eur. Phys. J. D 55, 319 (2009); O. J. Pike, F. Mackenroth, E. G. Hill, and S. J. Rose, Nat. Photon. 8, 434 (2014).

[7] G. V. Dunne and C. Schubert, Phys. Rev. D 72, 105004 (2005); H. Gies and K. Klingmuller, Phys. Rev. D 72, 065001 (2005); H. Gies and G. Torgrimsson, Phys. Rev. Lett. 116, 090406 (2016); I. A. Aleksandrov, G. Plunien, and V.M. Shabaev, Phys. Rev. D 94, 065024 (2016); 
I. A. Aleksandrov, G. Plunien, and V. M. Shabaev, Phys. Rev. D 96, 076006 (2017); C. Kohlfürst, arXiv:1708.08920.

[8] F. Hebenstreit, R. Alkofer, and H. Gies, Phys. Rev. Lett. 107, 180403 (2011).

[9] S. P. Kim and D. N. Page, Phys. Rev. D 75, 045013 (2007); H. Kleinert, R. Ruffini, and S. S. Xue, Phys. Rev. D 78, 025011 (2008); M. Ruf, G. R. Mocken, C. Muller, K. Z. Hatsagortsyan, and C. H. Keitel, Phys. Rev. Lett. 102, 080402 (2009); W. B. Han, R. Ruffini, and S. S. Xue, Phys. Lett. B 691, 99 (2010); F. Hebenstreit, J. Berges, and D. Gelfand, Phys. Rev. D 87, 105006 (2013); D. Berényi, S. Varró, V. V. Skokov, and P. Lévai, Phys. Lett. B 749, 210 (2015).

[10] C. Kohlfürst and R. Alkofer, Phys. Lett. B 756, 371 (2016).

[11] A. Ringwald, Phys. Lett. B 510, 107 (2001).

[12] D. M. Wolkow, Z. Phys. 94, 250 (1935).

[13] I. I. Goldman, Phys. Lett. 8, 103 (1964); A. I. Nikishov and V. I. Ritus, Sov. Phys. JETP 19, 529 (1964); T. W. B. Kibble, Phys. Rev. 138, B740 (1965).

[14] T. W. B. Kibble, Phys. Rev. 150, 1060 (1966).

[15] R. A. Neville and F. Rohrlich, Phys. Rev. D 3, 1692 (1971).

[16] I. Y. Dodin and N. J. Fisch, Phys. Rev. E 77, 036402 (2008).

[17] T. Heinzl, A. Ilderton, and M. Marklund, Phys. Lett. B 692 , 250 (2010).

[18] M. Lavelle and D. McMullan, Phys. Rev. D 91, 105022 (2015).

[19] Z. Huang and K.-J. Kim, Phys. Rev. ST Accel. Beams 10, 034801 (2007); B. W. J. McNeil and N. R. Thompson, Nat. Photonics 4, 814 (2010).

[20] C. Kohlfürst, H. Gies, and R. Alkofer, Phys. Rev. Lett. 112, 050402 (2014).

[21] Z. L. Li, D. Lu, and B.S. Xie, Phys. Rev. D 92, 085001 (2015).

[22] D. Bauer, P. Mulser, and W. H. Steeb, Phys. Rev. Lett. 75, 4622 (1995).

[23] C. Kohlfürst, Ph.D. Thesis, University of Graz, 2015.

[24] D. Vasak, M. Gyulassy, and H. T. Elze, Ann. Phys. (N.Y.) 173, 462 (1987); I. Bialynicki-Birula, P. Górnicki, and J. Rafelski, Phys. Rev. D 44, 1825 (1991); F. Hebenstreit, R. Alkofer, and H. Gies, Phys. Rev. D 82, 105026 (2010); F. Hebenstreit, R. Alkofer, and H. Gies, Phys. Rev. Lett. 107, 180403 (2011); F. Hebenstreit, Ph.D. Thesis, University of Graz, 2011.
[25] J. P. Boyd, Chebyshev and Fourier Spectral Methods, Dover Books on Mathematics (Dover, New York, 2001).

[26] W. H. Press, S. A. Teukolsky, W. T. Vetterling, and B. P. Flannery, Numerical Recipes in $\mathrm{C}++$ (Cambridge University Press, Cambridge, England, 2007).

[27] V. B. Krapchev, Phys. Rev. Lett. 42, 497 (1979); W. M. Manheimer, Phys. Fluids 28, 1569 (1985).

[28] F. Mackenroth and A. Di Piazza, Phys. Rev. A 83, 032106 (2011); F. Mackenroth and A. Di Piazza, Phys. Rev. A 85, 046102 (2012).

[29] T. W. B. Kibble, A. Salam, and J. A. Strathdee, Nucl. Phys. B96, 255 (1975); C. Harvey, T. Heinzl, A. Ilderton, and M. Marklund, Phys. Rev. Lett. 109, 100402 (2012).

[30] A. Otto, D. Seipt, D. Blaschke, B. Kämpfer, and S. A. Smolyansky, Phys. Lett. B 740, 335 (2015).

[31] E. Brezin and C. Itzykson, Phys. Rev. D 2, 1191 (1970); V. S. Popov, Zh. Eksp. Teor. Fiz. 63, 1586 (1972) [Sov. Phys. JETP 36, 840 (1973)]; N. B. Delone and V. P. Krainov, Multiphoton Processes in Atoms (Springer, Berlin, 1994); R. Kopold, W. Becker, M. Kleber, and G. G. Paulus, J. Phys. B 35, 217 (2002).

[32] S. A. Smolyansky, G. Ropke, S. M. Schmidt, D. Blaschke, V.D. Toneev, and A. V. Prozorkevich, arXiv:hep-ph/ 9712377; Y. Kluger, E. Mottola, and J. M. Eisenberg, Phys. Rev. D 58, 125015 (1998); S. M. Schmidt, D. Blaschke, G. Ropke, S. A. Smolyansky, A. V. Prozorkevich, and V. D. Toneev, Int. J. Mod. Phys. E 07, 709 (1998); J. C. R. Bloch, V. A. Mizerny, A. V. Prozorkevich, C. D. Roberts, S. M. Schmidt, S. A. Smolyansky, and D. V. Vinnik, Phys. Rev. D 60, 116011 (1999).

[33] P. B. Corkum, Phys. Rev. Lett. 71, 1994 (1993); G. G. Paulus, F. Grasbon, A. Dreischuh, H. Walther, R. Kopold, and W. Becker, Phys. Rev. Lett. 84, 3791 (2000); J. Liu, Classical Trajectory Perspective of Atomic Ionization in Strong Laser Fields: Semiclassical Modeling, Springer Briefs in Physics (Springer, Berlin, 2014).

[34] T. Nousch, D. Seipt, B. Kämpfer, and A. I. Titov, Phys. Lett. B 755, 162 (2016).

[35] Ph. A. Korneev, S. V. Popruzhenko, S. P. Goreslavski, T.-M. Yan, D. Bauer, W. Becker, M. Kübel, M. F. Kling, C. Rödel, M. Wünsche, and G. G. Paulus, Phys. Rev. Lett. 108, 223601 (2012). 